\title{
Fatores associados à infecção pelo HIV/AIDS entre adolescentes e adultos jovens matriculados em Centro de Testagem e Aconselhamento no Estado da Bahia, Brasil
}

\author{
Factors associated with HIV/AIDS infection \\ among adolescents and young adults enrolled in a Counseling \\ and Testing Center in the State of Bahia, Brazil
}

Bianca de Souza Pereira ${ }^{1}$

Maria Conceição Oliveira Costa ${ }^{1}$

Magali Teresópolis Reis Amaral ${ }^{1}$

Hervânia Santana da Costa ${ }^{1}$

Carlos Alberto Lima da Silva ${ }^{2}$

Vanessa Silva Sampaio ${ }^{2}$

\footnotetext{
${ }^{1}$ Departamento de Saúde, Universidade Estadual de Feira de Santana. Av. Transnordestina S/N Campus Universitário Módulo VI, Novo Horizonte. 44.031-460 Feira de Santana BA Brasil. bianca_enfermeira13@ hotmail.com ${ }^{2}$ Centro de Referência Estadual DST/HIV.
}

\begin{abstract}
The study seeks to investigate factors associated with HIV infection among adolescents and young adults registered in the Counseling and Testing Center (CTC) of Feira de Santana, Bahia. An analytical study with a cross-sectional design was conducted using data from the CTC Information System in the period from 2007 to 2011. Information on 3,768 individuals were studied and the proportional difference was checked using the Pearson chi-squared test and stratified analysis by Prevalence Rate $(P R)$ with confidence intervals of $95 \%$, considering the serological status as the outcome. During the period under study, 73 young people were infected with HIV (1.94\%). In the female population, statistically significant associations were identified for HIV status and drug use and consumption of alcohol and other drugs, as well as being married or in a stable relationship. In males, the consumption of other drugs and homosexual/bisexual orientation were associated with HIV status. In both sexes, in the category in a stable relationship the HIV status was associated with a partner using drugs and/or infected with a STD and/or HIV. These findings indicate the need for effective measures for the control and prevention of HIV infection among young people.
\end{abstract}

Key words AIDS, Adolescents, Young adults, Risk factors, Health vulnerability
Resumo O estudo procura investigar fatores associados à infecção pelo HIV entre adolescentes e adultos jovens, registrados no Centro de Testagem e Aconselhamento/CTA de Feira de Santana, Bahia. Estudo analítico com delineamento transversal, utilizando dados do Sistema de Informação do CTA (SI-CTA) no período de 2007 a 2011. Foram processadas informações de 3.768 indivíduos, sendo verificada a diferença de proporções, com Qui-quadrado de Pearson e análise estratificada, através da Razão de Prevalência/RP, com Intervalos de Confiança de 95\%, considerando como desfecho o status sorológico. No período estudado, 73 jovens estavam infectados pelo HIV (1,94\%). Na população feminina, foram identificadas associações, estatisticamente significantes, para soropositividade e consumo de drogas, consumo de bebidas alcoólicas e outras drogas; estar casada ou em união estável. No sexo masculino, associaram-se com a soropositividade o consumo de outras drogas e orientação homossexual/bissexual. Em ambos os sexos, na categoria com parceiro estável, a soropositividade mostrou associação com companheiro usuário de drogas elou portador de DST e/ou HIV. Esses achados sinalizam a necessidade de medidas eficazes no controle e prevenção da infecção pelo HIV, entre jovens.

Palavras-chave AIDS, Adolescente, Jovem, Fatores de risco, Vulnerabilidade em saúde 


\section{Introdução}

Segundo dados oficiais, em nível mundial, existem cerca de 34 milhões de pessoas vivendo com o vírus HIV-AIDS, cuja pandemia encontra-se em franca expansão, configurando-se num desafio aos diversos setores sociais, quanto às medidas de controle das susceptibilidades ligadas aos aspectos individuais e contextuais de exposição ao vírus ${ }^{1-3}$.

Estimativas apontam que, entre os infectados, mais de 10 milhões encontram-se na faixa etária entre 15 a 24 anos e a taxa de prevalência da população jovem apresenta tendência de aumento, configurando-se em importante problema saúde pública ${ }^{4,5}$.

No Brasil, face ao desafio de prevenir e controlar essa epidemia, autoridades governamentais e sociedade civil organizada mobilizam-se na busca de ampliar o conhecimento e a utilização de tecnologias que possam melhorar a qualidade de vida, investindo nas medidas de prevenção e de atenção aos indivíduos soropositivos ${ }^{6,7}$.

Nos aspectos relacionados à adolescência e juventude, indicadores dos diversos contextos apontam esses grupos como prioritários aos empreendimentos e estratégias de proteção e prevenção, face à alta susceptibilidade às doenças sexualmente transmissíveis ${ }^{8-10}$.

Assim, segundo dados do Ministério da Saúde, até final de 2012, do total das notificações de AIDS, 2.478 casos concentravam-se na faixa etária entre 10 a 14 anos, 12.246 , no grupo de 15 a 19 anos e 94.519 , entre 20 a 24 anos, totalizando 109.243 casos nas faixas compreendidas entre 10 a 24 anos, correspondendo a $16,6 \%$ da população geral acometida pelo vírus ${ }^{11}$.

A população adolescente apresenta baixa prevalência de soropositividade ao HIV, sendo o grupo mais precocemente acometido por via sexual direta. No entanto, os adultos jovens representam uma das faixas etárias de mais alta prevalência, cuja infecção pode ter ocorrido na adolescência, indicando prioridade absoluta dessa população, visto que a precocidade das medidas de atenção interfere com a evolução da infecção e doença. Os problemas vivenciados pelos portadores têm impacto decisivo no processo de integração social, em nível profissional, social, familiar e relações amorosas ${ }^{12-14}$.

No que se refere ao enfrentamento da AIDS nesta população, destaca-se como fator de exposição a prática sexual desprotegida ou uso descontinuado do preservativo, seja com parceiro estável ou eventual ${ }^{15}$. Entre jovens do sexo femi- nino, a atividade sexual desprotegida pode causar impacto sobre a vida reprodutiva, pelo conjunto de susceptibilidades relacionadas à contaminação, assim como problemas advindos da transmissão vertical ${ }^{16}$.

Assim, nos grupos populacionais com alto índice de vulnerabilidade o planejamento estratégico de ações demanda o reconhecimento dos aspectos culturais, sociais e econômicos em vista de compreender a dinâmica social e epidemiológica da infecção pelo vírus. Além disso, múltiplas mudanças integram o processo de socialização do jovem, incluindo o exercício da sexualidade e as possibilidades de exposição, como mudança de parceiro; pouca utilização do condom, na prática sexual; consumo frequente de álcool e outras substâncias psicoativas (SPA), entre outros, que contribuem para o aumento dos índices de contaminação pelo HIV $^{17,18}$.

A relevância do estudo está diretamente relacionada à importância do Centro de Referencia em DST/HIV/AIDS e do município sede para a população da região do semiárido e sertão do Estado da Bahia. Feira de Santana é a segunda maior cidade do Estado (cerca de 600 mil habitantes) ${ }^{19}$, localizada às margens de um importante entroncamento rodoviário (rodovias federais e estaduais), que faz ligação entre as regiões norte, nordeste e sudeste do país, destacando-se pela presença de um importante polo industrial do Estado.

Distando apenas $100 \mathrm{~km}$ da capital (Salvador) e na condição de portal do semiárido da Bahia, o município possui intenso fluxo migratório das populações pobres do sertão baiano. Em Feira de Santana está sediada a 2a Diretoria Regional de Saúde e o polo da microrregião de saúde, composto por mais 25 municípios do Estado, onde se destaca o Centro de Referência em DST/HIV/AIDS. Esse Centro de Referência, lotado em Feira de Santana, possui atuação estratégica para a região, funcionando em acordo ao plano de metas do Ministério da Saúde e Coordenação Estadual, no desenvolvimento das ações de prevenção, atendimento e controle dessas doenças, direcionadas à população dessa região. $\mathrm{O}$ Centro funciona através de suas unidades: Unidade de DST; Unidade/Centro de Testagem e Aconselhamento (CTA); Unidade de Assistência Especializada aos portadores de HIV/AIDS (SAE); Unidade de Assistência Farmacêutica; Unidade Laboratorial. $\mathrm{O}$ atendimento é direcionado às gestantes referenciadas pelo pré-natal da rede do SUS, para triagem da transmissão vertical; aos pacientes referenciados da rede básica do SUS, 
hospitais e policlínicas, assim como por demanda espontânea.

O objetivo deste estudo foi investigar os fatores associados à soropositividade ao HIV entre jovens matriculados no Centro de Testagem e Aconselhamento (CTA) do Centro de Referência Municipal (CRM) de DST/HIV/AIDS de Feira de Santana, Bahia, Brasil.

\section{Método}

Estudo observacional, com delineamento transversal, realizado com adolescentes e jovens, de ambos os sexos, na faixa de 13 a 24 anos, que realizaram testagem sorológica para HIV, no Centro de Referência Municipal em DST/HIV/ AIDS de Feira de Santana, no período compreendido entre 2007 a 2011.

O Centro de Testagem e Aconselhamento (CTA) em DST/HIV/AIDS de Feira de Santana possui estrutura material e de recursos humanos para oferecer atenção integral aos usuários, referenciados do Sistema Único de Saúde (SUS) ou de demanda espontânea, segundo critérios do Ministério da Saúde do Brasil. Nos casos de sorologia positiva, o usuário é encaminhado para o Serviço de Assistência Especializada/SAE, Hospital Dia/HD e Convencional/HC e Assistência Domiciliar Terapêutica/ADT, que funcionam com o Sistema de Saúde Regional, integrando o Centro de Referência Municipal/CRM.

Os dados foram coletados no Sistema de Informação do Centro de Testagem e Aconselhamento do (SI-CTA) do CRM, utilizando um formulário específico, com registro manual das variáveis pertinentes aos objetivos do estudo.

A variável de desfecho foi status sorológico, categorizada como soropositivo ou soronegativo. As demais variáveis foram organizadas em grupos: 1- variáveis sociodemográficas: sexo (feminino e masculino), faixa etária (13-24 anos), estado civil (casado/união estável; solteiro) escolaridade (<8anos ou $>$ 8anos), cor da pele, ocupação, município de residência (Feira de Santana e outros) e zona de residência (rural ou urbana); 2- variáveis comportamentais e clínicas: presença de DST; tratamento da doença sexualmente transmissível/DST (serviço de saúde, automedicação); multiplicidade de parceiros; orientação sexual (heterossexual, homo/bissexual); uso de drogas; uso de álcool; uso de outras drogas; tipo de exposição ao HIV (relação sexual, outras); parceiro estável (usuário de drogas e/ou portador de DST/HIV, outros); 3- Variáveis relaciona- das ao uso de preservativos, segundo parceiro estável ou eventual: uso de preservativo e motivo para não utilização (fatores relacionados ao parceiro, fatores externos, fatores pessoais).

Os dados foram processados no programa Epidata versão 3.1 e as análises realizadas no programa SPSS versão 16.0. Para fins de análise, adolescentes (13 a 19) e adultos jovens (20 a 24 anos) foram agrupados segundo o sexo (masculino e feminino) e subdivididos de acordo com o status sorológico (soropositivo e soronegativo). Em seguida, foram calculadas frequências simples, estimando-se as proporções dos indivíduos soropositivos e soronegativos, segundo o sexo e por categoria de variável.

As diferenças de proporções foram testadas quanto à significância estatística por meio do Qui-quadrado $(\chi 2)$ de Pearson, adotando-se nível de significância $\mathrm{p}<0,05$. Para avaliar possíveis associações entre fatores de exposição e status sorológico, de acordo com sexo, procedeu-se a análise estratificada das variáveis agrupadas, sendo calculadas as Razões de Prevalência (RP) e os respectivos Intervalos de Confiança (IC 95\%). Este projeto foi aprovado pelo Comitê de Ética em Pesquisa da Universidade Estadual de Feira de Santana.

\section{Resultados}

No período entre 2007 a 2011, o Centro de Testagem e Aconselhamento do Centro de Referência Municipal em DST/HIV/AIDS de Feira de Santana registrou um total de 20.332 atendimentos, dos quais $558(2,7 \%)$ apresentaram soropositividade para o HIV, sendo 321 do sexo masculino e 237 do sexo feminino. No presente estudo, foram investigados dados de todos os 3.768 indivíduos atendidos no CRM que estavam na faixa etária de 13 a 24 anos sendo $73,1 \%$ do sexo feminino e $26,9 \%$ do sexo masculino, correspondendo a $18,5 \%$ do total de usuários. Na avaliação do status sorológico, verificou-se prevalência de soropositividade de $1,94 \%$ na população geral de adolescentes e jovens, sendo $3,0 \%$ no sexo masculino e $1,6 \%$ no sexo feminino.

Os dados sociodemográficos dos indivíduos de ambos os sexos, independente do status sorológico, mostraram características semelhantes, com a maioria do sexo feminino $(73,1 \%)$; cor da pele parda (70-80\%); oito ou mais anos de escolaridade (60-80\%), ocupações diversas (40-67\%), residentes em Feira de Santana (83-97\%) e na zona urbana (95-100\%). 
Na Tabela 1 verifica-se que há divergência do status sorológico entre os sexos, quanto à situação conjugal, com as jovens mostrando maior proporção de indivíduos soropositivos entre as casadas ou com união estável ( $\mathrm{p}<0,05)$. Notase ainda que há mais jovens soropositivos de ambos os sexos dentre aqueles residentes em Feira de Santana $(\mathrm{p}<0,05)$.

Tabela 1. Características sociodemográficas, segundo sexo dos adolescentes e adultos jovens de acordo com o status sorológico matriculados no Centro de Testagem / CTA do Programa Municipal de DST/HIV/ AIDS, Feira de Santana, Bahia, (2007-2011).

\begin{tabular}{|c|c|c|c|c|c|}
\hline \multirow{3}{*}{$\begin{array}{c}\text { Características } \\
\text { sociodemográficas }\end{array}$} & \multicolumn{4}{|c|}{ Masculino } & \multirow{3}{*}{$\begin{array}{l}\text { Teste qui-quadrado } \\
\qquad\left(\chi^{2}\right)^{\mathrm{a}}\end{array}$} \\
\hline & \multicolumn{2}{|c|}{ Soronegativos } & \multicolumn{2}{|c|}{ Soropositivos } & \\
\hline & $\mathbf{N}$ & $\%$ & $\mathbf{N}$ & $\%$ & \\
\hline \multicolumn{6}{|l|}{ Estado civil } \\
\hline Casado (a)\União estável & 144 & 15 & 6 & 21,4 & 0,351 \\
\hline Não casado & 815 & 85 & 22 & 78,6 & \\
\hline \multicolumn{6}{|l|}{ Escolaridade } \\
\hline$<8$ anos & 299 & 31,5 & 5 & 17,9 & 0,124 \\
\hline$\geq 8$ anos & 650 & 68,5 & 23 & 82,1 & \\
\hline \multicolumn{6}{|l|}{ Cor da pele } \\
\hline Pardo (a) & 715 & 74,2 & 20 & 69 & 0,529 \\
\hline Outros & 249 & 25,8 & 9 & 31 & \\
\hline \multicolumn{6}{|l|}{ Ocupação } \\
\hline Estudante & 421 & 44,6 & 10 & 33,3 & 0,223 \\
\hline Outros & 524 & 55,4 & 20 & 66,7 & \\
\hline \multicolumn{6}{|l|}{ Município de residência } \\
\hline Feira de Santana & 937 & 95,1 & 26 & 86,7 & $0,038^{*}$ \\
\hline Outros municípios da região & 48 & 4,9 & 4 & 13,3 & \\
\hline \multicolumn{6}{|l|}{ Zona de Residência } \\
\hline Rural & 21 & 2,1 & 0 & 0 & 0,419 \\
\hline Urbana & 964 & 97,9 & 30 & 100 & \\
\hline
\end{tabular}

\begin{tabular}{|c|c|c|c|c|c|}
\hline \multirow{3}{*}{$\begin{array}{c}\text { Características } \\
\text { sociodemográficas }\end{array}$} & \multicolumn{4}{|c|}{ Feminino } & \multirow{3}{*}{$\begin{array}{l}\text { Teste qui-quadrado } \\
\qquad\left(\chi^{2}\right)^{\mathrm{a}}\end{array}$} \\
\hline & \multicolumn{2}{|c|}{ Soronegativos } & \multicolumn{2}{|c|}{ Soropositivos } & \\
\hline & $\mathbf{N}$ & $\%$ & $\mathbf{N}$ & $\%$ & \\
\hline \multicolumn{6}{|l|}{ Estado civil } \\
\hline Casado (a)\União estável & 1153 & 44,3 & 26 & 61,9 & \multirow[t]{2}{*}{$0,023^{*}$} \\
\hline Não casado & 1451 & 55,7 & 16 & 38,1 & \\
\hline \multicolumn{6}{|l|}{ Escolaridade } \\
\hline$<8$ anos & 1013 & 39,3 & 20 & 50 & \multirow[t]{2}{*}{0,169} \\
\hline$\geq 8$ anos & 1566 & 60,7 & 20 & 50 & \\
\hline \multicolumn{6}{|l|}{ Cor da pele } \\
\hline Pardo (a) & 2043 & 78,1 & 36 & 87,8 & \multirow[t]{2}{*}{0,134} \\
\hline Outros & 574 & 21,9 & 5 & 12,2 & \\
\hline \multicolumn{6}{|l|}{ Ocupação } \\
\hline Estudante & 1575 & 60,8 & 19 & 50 & \multirow[t]{2}{*}{0,176} \\
\hline Outros & 1015 & 39,2 & 19 & 50 & \\
\hline \multicolumn{6}{|l|}{ Município de residência } \\
\hline Feira de Santana & 2626 & 96,9 & 36 & 83,7 & \multirow[t]{2}{*}{$0,000^{*}$} \\
\hline Outros municípios da região & 84 & 3,1 & 7 & 16,3 & \\
\hline \multicolumn{6}{|l|}{ Zona de Residência } \\
\hline Rural & 137 & 5,1 & 0 & 0 & \multirow[t]{2}{*}{0,130} \\
\hline Urbana & 2573 & 94,9 & 43 & 100 & \\
\hline
\end{tabular}

Fonte: SI- CTA Feira de Santana. ${ }^{\text {a }}$ Teste qui-quadrado $\left(\chi^{2}\right)$ de Pearson para diferenças de proporções entre as categorias das variáveis do estudo e status sorológicos; ${ }^{\mathrm{b}} \mathrm{p}<0,05$ (estatisticamente significante). 
As estimativas de Razão de Prevalência (RP) relacionadas aos fatores de exposição (Tabela 2) mostraram resultados significantes para ambos os sexos, na categoria "uso de outras drogas", onde o grupo soropositivo apresentou alta prevalência, no sexo masculino ( $R P=13,2 \%$ - IC 5,12$34,28)$ e no feminino ( $\mathrm{RP}=6,7$; IC 3,67-12,28).

Entre jovens do sexo masculino, verificou-se prevalência mais elevada de indivíduos soropositivos no grupo homossexual e bissexual ( $\mathrm{P}=$ $9,9 \%)$ em relação ao heterossexual $(\mathrm{P}=1,9 \%)$, com $\mathrm{RP}=5,21$ e IC 2,57-10,57.

No sexo feminino, constatou-se que aquelas soropositivas têm ocorrência mais elevada de "uso de drogas" ( $\mathrm{RP}=2,1$ e IC 1,15-3,82), "uso de álcool" ( $\mathrm{RP}=2,1$ e IC 1,16- 3,91) e "casadas ou em união estável" ( $\mathrm{RP}=$ 2,02 e IC 1,09-3,75).

Ainda em relação aos resultados da Tabela 2, vale salientar que, para ambos os sexos, a maioria dos indivíduos negou o acometimento por DST. Entretanto, no sexo masculino, a proporção entre os jovens soropositivos foi três vezes maior (70\%), em relação aos soronegativos $(21,3 \%)$; enquanto que, no sexo feminino a tendência é oposta: a frequência dessas doenças no grupo soronegativo foi superior $(12,6 \%)$, comparado ao soropositivo (7,0\%).

A maioria dos indivíduos de ambos os sexos, independente do status sorológico, relatou tratamento de DST em serviço de saúde e a presença

Tabela 2. Fatores de exposição à infecção pelo HIV, segundo o sexo, de adolescentes e adultos jovens matriculados no Centro de Testagem / CTA do Programa Municipal de DST/HIV/AIS, Feira de Santana, Bahia, (2007-2011).

\begin{tabular}{|c|c|c|c|c|c|c|c|}
\hline \multirow{3}{*}{ Fatores de exposição } & \multicolumn{4}{|c|}{ Masculino } & \multirow{3}{*}{ Prevalência } & \multirow{3}{*}{$\mathbf{R P}$} & \multirow{3}{*}{ IC } \\
\hline & \multicolumn{2}{|c|}{ Soronegativos } & \multicolumn{2}{|c|}{ Soropositivos } & & & \\
\hline & $\mathbf{N}$ & $\%$ & $\mathbf{N}$ & $\%$ & & & \\
\hline \multicolumn{8}{|l|}{ Escolaridade } \\
\hline$<8$ & 299 & 31,5 & 5 & 17,9 & 1,6 & 0,48 & $0,18-1,25$ \\
\hline$\geq 8$ & 650 & 68,5 & 23 & 82,1 & 3,4 & & \\
\hline \multicolumn{8}{|l|}{ Estado civil } \\
\hline Casado (a)\União estável & 144 & 15 & 6 & 21,4 & 4 & 1,52 & $0,62-3,69$ \\
\hline Não casado & 815 & 85 & 22 & 78,6 & 2,6 & & \\
\hline \multicolumn{8}{|l|}{ Presença de DST } \\
\hline Não & 775 & 78,7 & 21 & 30 & 2,6 & & \\
\hline Sim & 210 & 21,3 & 9 & 70 & 4,1 & 1,55 & $0,32-3,35$ \\
\hline \multicolumn{8}{|l|}{ Tratamento da DST } \\
\hline Serviço de Saúde & 162 & 80,2 & 5 & 62,5 & 2,99 & 0,42 & $0,11-1,72$ \\
\hline Não tratou/automedicação & 40 & 19,8 & 3 & 37,5 & 6,98 & & \\
\hline \multicolumn{8}{|l|}{ Multiplicidade de parceiros } \\
\hline Não & 64 & 34,8 & 6 & 37,5 & 8,6 & & \\
\hline Sim & 120 & 65,2 & 10 & 62,5 & 7,7 & 0,89 & $0,34-2,33$ \\
\hline \multicolumn{8}{|l|}{ Uso de drogas } \\
\hline Não & 406 & 41,3 & 10 & 33,3 & 2,4 & & \\
\hline Sim & 578 & 58,7 & 20 & 66,7 & 3,3 & 1,39 & $0,65-2,24$ \\
\hline \multicolumn{8}{|l|}{ Uso de álcool } \\
\hline Não & 464 & 47,1 & 12 & 40 & 2,5 & & \\
\hline Sim & 521 & 52,9 & 18 & 60 & 3,3 & 1,32 & $0,64-2,72$ \\
\hline \multicolumn{8}{|l|}{ Uso de outras drogas } \\
\hline Não & 732 & 25,7 & 5 & 16,7 & 0,7 & & \\
\hline Sim & 253 & 74,3 & 25 & 83,3 & 9 & 13,25 & $5,12-34,28^{\star}$ \\
\hline \multicolumn{8}{|l|}{ Exposição ao HIV } \\
\hline Relação sexual & 942 & 97,5 & 29 & 96,7 & 3 & 0,74 & $0,10-5,26$ \\
\hline Outras ${ }^{a}$ & 24 & 2,5 & 1 & 3,3 & 4 & & \\
\hline \multicolumn{8}{|l|}{ Orientação sexual } \\
\hline Heterossexual & 85,9 & 1,9 & 15 & 51,7 & 1,9 & & \\
\hline Homossexual/bissexual & 14,1 & 9,9 & 14 & 48,3 & 9,9 & 5,21 & $2,57-10-57^{*}$ \\
\hline
\end{tabular}


Tabela 2. continuação

\begin{tabular}{|c|c|c|c|c|c|c|c|}
\hline \multirow{3}{*}{ Fatores de exposição } & \multicolumn{4}{|c|}{ Feminino } & \multirow{3}{*}{ Prevalência } & \multirow{3}{*}{$\mathbf{R P}$} & \multirow{3}{*}{ IC } \\
\hline & \multicolumn{2}{|c|}{ Soronegativos } & \multicolumn{2}{|c|}{ Soropositivos } & & & \\
\hline & $\mathbf{N}$ & $\%$ & $\mathrm{~N}$ & $\%$ & & & \\
\hline \multicolumn{8}{|l|}{ Escolaridade } \\
\hline$<8$ & 1013 & 39,3 & 20 & 50 & 1,9 & 1,53 & $0,83-2,83$ \\
\hline$\geq 8$ & 1566 & 60,7 & 20 & 50 & 1,3 & & \\
\hline \multicolumn{8}{|l|}{ Estado civil } \\
\hline Casado (a)\União estável & 1153 & 44,3 & 26 & 61,9 & 2,2 & 2,02 & $1,09-3,75$ \\
\hline Não casado & 1451 & 55,7 & 16 & 38,1 & 1,1 & & \\
\hline \multicolumn{8}{|l|}{ Presença de DST } \\
\hline Não & 2368 & 87,4 & 40 & 93 & 1,7 & & \\
\hline Sim & 342 & 12,6 & 3 & 7 & 0,9 & 0,52 & $016-1,68$ \\
\hline \multicolumn{8}{|l|}{ Tratamento da DST } \\
\hline Serviço de Saúde & 295 & 87,3 & 2 & 66,7 & 0,67 & 0,29 & $0,03-3,2$ \\
\hline Não tratou/automedicação & 43 & 12,7 & 1 & 33,3 & 2,27 & & \\
\hline \multicolumn{8}{|l|}{ Multiplicidade de parceiros } \\
\hline Não & 1957 & 77,4 & 31 & 27,9 & 1,6 & & \\
\hline Sim & 573 & 22,6 & 12 & 72,1 & 2,1 & 1,31 & $0,67-2,54$ \\
\hline \multicolumn{8}{|l|}{ Uso de drogas } \\
\hline Não & 2020 & 74,7 & 25 & 58,1 & 1,2 & & \\
\hline Sim & 683 & 25,3 & 18 & 41,9 & 2,6 & 2,1 & $1,15-3,82$ \\
\hline \multicolumn{8}{|l|}{ Uso de álcool } \\
\hline Não & 2082 & 76,8 & 26 & 60,5 & 1,2 & & \\
\hline Sim & 628 & 23,2 & 17 & 39,5 & 2,6 & 2,13 & $1,16-3,91$ \\
\hline \multicolumn{8}{|l|}{ Uso de outras drogas } \\
\hline Não & 2503 & 92,4 & 27 & 35,3 & 1 & & \\
\hline Sim & 207 & 7,6 & 16 & 62,8 & 6,7 & 6,72 & $3,67-12,28$ \\
\hline \multicolumn{8}{|l|}{ Exposição ao HIV } \\
\hline Relação sexual & 2616 & 97,7 & 43 & 100 & 1,6 & - & - \\
\hline Outras ${ }^{a}$ & 61 & 2,3 & - & - & - & & \\
\hline \multicolumn{8}{|l|}{ Orientação sexual } \\
\hline Heterossexual & 2551 & 98,7 & 43 & 100 & 1,7 & & \\
\hline Homossexual/bissexual & 33 & 1,3 & - & - & - & - & - \\
\hline
\end{tabular}

Fonte: SI- CTA/ Feira de Santana. ${ }^{*}$ intervalo de confiança de 95\% (estatisticamente significante). ${ }^{a}$ transfusão de sangue, compartilhamento de seringas e agulhas, ocupacional, transmissão vertical, anfetaminas, violência e/ou abuso sexual, outros ( masculino $\mathrm{n}=25)$, (feminino $\mathrm{n}=61)$.

de múltiplos parceiros; entretanto, vale ressaltar altas proporções (acima de 30\%) da automedicação entre os soropositivos, assim como multiplicidade de parceiros no grupo soropositivo do sexo feminino, comparado ao soronegativo (72,1\% e 22,6\%, respectivamente).

As análises de Razão de Prevalência (RP) para a utilização do preservativo com parceiro estável ou eventual (Tabela 3) mostraram baixa frequência dessa prática em ambos os sexos, independente do status sorológico. Contudo, em ambos os sexos, na presença de união estável, foi observada associação significante entre soropositividade e parceiro usuário de drogas ou portador de DST/HIV, com ocorrência de infecção cinco vezes superior nesse grupo, em relação aos soronegativos, sendo a prevalência de $11,5 \%$, no sexo masculino e $8,3 \%$, no feminino.

No sexo feminino, os resultados significantes apontaram entre aquelas soropositivas há ocorrência maior da não utilização do preservativo motivada por fatores externos (RP 6,07 e IC 1,38$26,65)$ e por algum motivo pessoal (RP 2,27 e IC $1,09-4,71)$, quando comparadas às soronegativas.

\section{Discussão}

Nesta investigação, realizada com jovens, na maioria do sexo feminino, cor da pele parda, re- 
Tabela 3. Fatores de exposição à infecção pelo HIV, segundo o sexo, de adolescentes e adultos jovens matriculados no Centro de Testagem / CTA do Programa Municipal de DST/HIV/AIS, Feira de Santana, Bahia, (2007-2011).

\begin{tabular}{|c|c|c|c|c|c|c|c|}
\hline \multirow{3}{*}{ Tipo de parceiro e variáveis } & \multicolumn{4}{|c|}{ Masculino } & \multirow{3}{*}{ Prevalência } & \multirow{3}{*}{ RP } & \multirow{3}{*}{ IC } \\
\hline & \multicolumn{2}{|c|}{ Soronegativos } & \multicolumn{2}{|c|}{ Soropositivos } & & & \\
\hline & $\mathbf{N}$ & $\%$ & $\mathbf{N}$ & $\%$ & & & \\
\hline \multicolumn{8}{|l|}{ Parceiro estável } \\
\hline \multicolumn{8}{|l|}{ Uso do preservativo } \\
\hline Não & 501 & 82,8 & 16 & 88,9 & 3,1 & & \\
\hline Sim & 104 & 17,2 & 2 & 11,1 & 1,9 & 1,64 & $0,38-7,02$ \\
\hline \multicolumn{8}{|l|}{$\begin{array}{l}\text { Motivo para não uso do } \\
\text { preservativo }\end{array}$} \\
\hline $\begin{array}{l}\text { Fatores relacionados ao } \\
\text { parceiro }\end{array}$ & 167 & 35,5 & 6 & 40 & 3,5 & & \\
\hline Fatores externos & 4 & 0,9 & - & - & - & - & - \\
\hline Fatores pessoais & 299 & 63,6 & 9 & 60 & 2,9 & 0,84 & $0,30-2,32$ \\
\hline \multicolumn{8}{|l|}{ Grupo do parceiro fixo } \\
\hline $\begin{array}{l}\text { Usuário de drogas e/ou } \\
\text { portador de DST e/ou HIV }\end{array}$ & 23 & 7,2 & 3 & 30 & 11,5 & 5,01 & $1,37-18,23$ \\
\hline Outros & 297 & 92,8 & 7 & 70 & 2,3 & & \\
\hline \multicolumn{8}{|l|}{ Parceiro eventual } \\
\hline \multicolumn{8}{|l|}{ Uso do preservativo } \\
\hline Não & 412 & 66,7 & 14 & 87,5 & 3,3 & & \\
\hline Sim & 206 & 33,3 & 2 & 12,5 & 1 & 3,41 & $0,78-14,89$ \\
\hline \multicolumn{8}{|l|}{$\begin{array}{l}\text { Motivo para não uso do } \\
\text { preservativo }\end{array}$} \\
\hline $\begin{array}{l}\text { Fatores relacionados ao } \\
\text { parceiro }\end{array}$ & 74 & 19,3 & 1 & 8,3 & 1,3 & & \\
\hline Fatores externos & 2 & 0,5 & - & - & - & - & - \\
\hline Fatores pessoais & 307 & 80,2 & 11 & 91,7 & 3,5 & 2,59 & $0,34-19,78$ \\
\hline
\end{tabular}

sidentes em Feira de Santana e heterossexuais, verificou-se que $1,26 \%$, na faixa entre 13 até 19 anos e $2,19 \%$ entre 20 a 24 anos apresentaram soropositividade para o HIV. Em nível mundial, estudos têm demonstrado tendência do aumento das taxas de infecção na população de jovens, independente do contexto socioeconômico ${ }^{5,20}$. Assinala-se ainda que adolescentes (13-19 anos) representa o grupo mais precocemente acometido por via sexual direta. Enquanto que, os adultos jovens (20-24 anos) representam uma das faixas etária de maior prevalência da infecção pelo HIV, possivelmente adquirida na fase da adolescência. Isto evidencia a importância da população de 13-24 anos no direcionamento das estratégias de proteção, prevenção e controle ${ }^{21}$.

No período de estudo, a maioria dos indivíduos era solteiro, cabendo ressaltar a associação significante entre soropositividade e situação conjugal estável, entre jovens do sexo feminino. Esse importante achado está de acordo com estudos anteriores que citam os fenômenos de feminização e heterossexualização da AIDS, visto que mulheres em união estável ou casadas designam confiança no parceiro, não utilizam o preservativo ou fazem de forma descontinuada. Pesquisas apontam aumento da infecção pelo vírus HIV entre mulheres heterossexuais, pobres, com pouca escolaridade, negras ou pardas, com parceiro único e histórico de múltiplas violências, demonstrando a complexidade epidemiológica desta questão e evidenciando que o contexto social potencializa a vulnerabilidade ao vírus ${ }^{22}$.

No que concerne à escolaridade, verificou-se nesse estudo uma alta proporção de indivíduos com baixa escolaridade e a maioria exercendo ocupações diversas da prática estudantil. Entretanto, no sexo feminino, o grupo soropositivo apresentou a maior frequência de indivíduos com baixa escolaridade. Estes achados concordam com 
Tabela 3. continuação

\begin{tabular}{|c|c|c|c|c|c|c|c|}
\hline \multirow{3}{*}{ Tipo de parceiro e variáveis } & \multicolumn{4}{|c|}{ Feminino } & \multirow{3}{*}{ Prevalência } & \multirow{3}{*}{$\mathbf{R P}$} & \multirow{3}{*}{ IC } \\
\hline & \multicolumn{2}{|c|}{ Soronegativos } & \multicolumn{2}{|c|}{ Soropositivos } & & & \\
\hline & $\mathbf{N}$ & $\%$ & $\mathbf{N}$ & $\%$ & & & \\
\hline \multicolumn{8}{|l|}{ Parceiro estável } \\
\hline \multicolumn{8}{|l|}{ Uso do preservativo } \\
\hline Não & 2224 & 92,8 & 37 & 90,2 & 1,6 & & \\
\hline Sim & 172 & 7,2 & 4 & 9,8 & 2,3 & 0,72 & $0,25-1,99$ \\
\hline \multicolumn{8}{|l|}{$\begin{array}{l}\text { Motivo para não uso do } \\
\text { preservativo }\end{array}$} \\
\hline $\begin{array}{l}\text { Fatores relacionados ao } \\
\text { parceiro }\end{array}$ & 993 & 47,3 & 10 & 27 & 1 & & \\
\hline Fatores externos & 31 & 1,5 & 2 & 5,4 & 6,1 & 6,07 & $1,38-26,65$ \\
\hline Fatores pessoais & 1076 & 51,2 & 25 & 67,6 & 2,3 & 2,27 & $1,09-4,71$ \\
\hline \multicolumn{8}{|l|}{ Grupo do parceiro fixo } \\
\hline $\begin{array}{l}\text { Usuário de drogas e/ou } \\
\text { portador de DST e/ou HIV }\end{array}$ & 77 & 6,5 & 7 & 30,4 & 8,3 & 5,81 & $2,46-13,74$ \\
\hline Outros $^{\mathrm{a}}$ & 1101 & 93,5 & 16 & 69,6 & 1,4 & & \\
\hline \multicolumn{8}{|l|}{ Parceiro eventual } \\
\hline \multicolumn{8}{|l|}{ Uso do preservativo } \\
\hline Não & 245 & 59,8 & 5 & 62,5 & 2 & & \\
\hline Sim & 165 & 40,2 & 3 & 37,5 & 1,8 & 1,12 & $0,27-4,60$ \\
\hline \multicolumn{8}{|l|}{$\begin{array}{l}\text { Motivo para não uso do } \\
\text { preservativo }\end{array}$} \\
\hline $\begin{array}{l}\text { Fatores relacionados ao } \\
\text { parceiro }\end{array}$ & 57 & 26,5 & 1 & 20 & 1,7 & & \\
\hline Fatores externos & 24 & 11,2 & - & - & - & - & - \\
\hline Fatores pessoais & 134 & 62,3 & 4 & 80 & 2,9 & 1,68 & $0,19-14,72$ \\
\hline
\end{tabular}

Fonte: SI- CTA/ Feira de Santana. * intervalo de confiança de 95\% (estatisticamente significante); a transfusão de sangue, hemofílico e outros (masculino $\mathrm{n}=304)$, (feminino, $\mathrm{n}=1117$ ).

a literatura mundial, que mostra modificações no padrão de transmissão do HIV, em decorrência do baixo nível de formação escolar, sugerindo a possível relação entre pauperização populacional e infecção ou doença. A disseminação do HIV junto às camadas mais pobres e com baixo nível de escolaridade aponta maior susceptibilidade deste grupo, possivelmente pela falta de informações e menor acesso aos meios de prevenção ${ }^{12,23}$.

Os dados da presente pesquisa mostraram maior frequência de soropositivos entre indivíduos do sexo feminino, possivelmente, pelo maior volume de atendimentos de mulheres. Esses achados ratificam pesquisas que mostram alta proporção de atendimentos do sexo feminino nos centros de referência em DST/HIV/AIDS ${ }^{24,25}$, com concomitante aumento da incidência de infecção pelo HIV, verificada nos últimos anos, fenômeno conhecido como feminização da AIDS ${ }^{20,24-27}$. Pesquisas realizadas em diversos contextos ${ }^{23,28}$ têm mostrado que mulheres procuram com maior frequência os serviços de Saúde e Centros de Re- ferência, seja para buscar ajuda pessoal aos problemas de saúde, em geral, para a realização do pré-natal ou resolver problemas relacionados à gestação e respectivos filhos.

O discurso da feminização da AIDS envolve questões biológicas e socioculturais. As relações de gênero, as condições de vida desfavoráveis e o contexto sociocultural em que vivem contribuem para a transmissão sexual do $\mathrm{HIV}^{29,30}$. Segundo estudos, independente do contexto, a maioria das mulheres infectadas apresenta baixa escolaridade e nível ocupacional (prendas do lar) e são expostas ao vírus, principalmente através de relações heterossexuais com companheiros de união estável ${ }^{29}$.

Os achados do presente estudo ratificam outras investigações, onde foram observadas altas prevalências da não utilização do preservativo entre jovens do sexo feminino e com união estável. Além disso, os indivíduos do sexo feminino, com união estável, cujos parceiros eram usuários de droga ou portadores de DST/HIV, apre- 
sentaram associação significante no grupo soropositivo. Segundo estudos, este comportamento sexual feminino pode estar ligado às questões de gênero e poder, do masculino sobre o feminino, pela condição de submissão, assim como questões morais, preconceitos e tabus, relacionados à sexualidade feminina, as quais são expressões da submissão e falta de autonomia feminina ${ }^{25,31}$.

Pesquisas relacionadas às questões de gênero demonstram a susceptibilidade da mulher, frente à dominação do homem, identificando o masculino como razão, regulação, liberdade do exercício sexual, enquanto a mulher marcada pelo conformismo, dificuldade de negociação ao uso do preservativo, situações que geram violências e susceptibilidades ${ }^{25,31,32}$. A abordagem das questões de gênero requer o enfoque relacional e transversal, que inclui a interação entre classe social, etnia/raça, diferença de geração, cultura, escolaridade, para a compreensão das questões de poder e exigências que se estabelecem entre homens e mulheres, no exercício das respectivas sexualidades, principalmente entre adolescentes e adultos jovens ${ }^{33}$. A compreensão da magnitude e transcendência desses aspectos é de fundamental importância para a implementação de políticas e ações intersetoriais de educação, saúde e integração social ${ }^{34}$. Em nível nacional, as ações programáticas do Ministério da Saúde direcionadas ao combate e controle da epidemia da AIDS englobam questões relacionadas aos direitos humanos, permitindo analisar a situação de vulnerabilidade ao HIV, no plano individual, social e programático, levando em conta as questões de gênero e poder, sexismo e homofobia ${ }^{35}$.

$\mathrm{Na}$ presente pesquisa, a relação sexual foi a principal forma de transmissão do HIV, destacando-se a alta frequência da não utilização ou uso eventual do preservativo, na prática sexual dos jovens que participaram deste estudo. O grupo soropositivo de ambos os sexos apresentou baixa proporção na utilização do preservativo, concordando com outras pesquisas ${ }^{15}$. Além disso, foram verificadas associações estatisticamente significantes entre soropositividade e o uso do preservativo, com interferência de fatores ambientais e pessoais. Estes resultados assemelham-se aos achados de estudos realizados nas diversas regiões do país, onde foi observado que, cerca de 30\% dos indivíduos que não utilizavam o preservativo, justificaram não dispor de um no momento da relação e $30 \%$ não priorizando esta prática. Nesses estudos observou-se que, apesar de haver conhecimento declarado nas técnicas de utilização do preservativo, isso não refletiu em uso habitual ${ }^{17,36}$.
Em Feira de Santana, apesar da maior exposição heterossexual, conforme resultado da presente pesquisa, foi detectada associação estatisticamente significante entre soropositividade e comportamento homo ou bissexual, entre os indivíduos do sexo masculino, resultado que corroboram com outros estudos ${ }^{37}$. $\mathrm{O}$ aumento dos índices de infecção pelo HIV nesse grupo tem sido apontado por vários estudiosos, o que sugere susceptibilidade, especialmente entre indivíduos jovens ${ }^{5}$. Entretanto, vale enfatizar que, independente da orientação sexual, a prática sexual desprotegida e a multiplicidade de parceiros, somadas ao uso de álcool e drogas são fatores de impacto para o aumento da incidência e prevalência da infecção pelo HIV, demonstrando a importância das ações de prevenção ${ }^{38}$. Esta realidade reforça a necessidade das estratégias direcionadas aos grupos susceptíveis, visando à redução de danos e mudança de atitudes frente à exposição ao vírus e, consequentemente, diminuição da expansão dessa infecção e diminuição do impacto epidemiológico provocado na saúde da população, principalmente em grupos mais vulneráveis ${ }^{26,37}$.

Ainda entre os fatores de exposição, a literatura aponta a utilização de drogas lícitas e ilícitas como um dos riscos e susceptibilidade ao HIV. No atual estudo ficou evidenciado, no sexo feminino, associação estatisticamente significante entre soropositividade e uso de álcool, uso de drogas, assim como, em ambos os sexos, houve significância estatística entre infecção pelo HIV, DST e parceiro usuário de droga e soropositivos, corroborando com outras pesquisas que também demonstram achados semelhantes ${ }^{18}$.

Em Feira de Santana foi observada maior prevalência de DST em indivíduos de 13-24 anos soropositivos para o HIV. As DST são consideradas como importantes fatores de risco para a infecção pelo HIV. Nos Estados Unidos estimase cerca de 19 milhões de indivíduos portadores de DST, com cerca de 50\% na faixa-etária entre 15 a 24 anos $^{39}$. Entre os determinantes para manutenção da transmissão das DST e sua alta vulnerabilidade entre jovens destacam-se a pouca utilização ou o uso descontinuado do preservativo, a baixa escolaridade, a variabilidade ou multiplicidade de parceiros, a imaturidade psicossocial (sentimentos de onipotência) e a não adesão aos métodos preventivos ${ }^{12}$.

Nesta investigação foi verificado que, apesar da maior parte dos jovens ter realizado tratamento para as DST em Serviços de Saúde, parte significativa destes se automedicou. Esta atitude costuma estar relacionada com o aumento da 
resistência antimicrobiana, quadros subclínicos que mantêm a cadeia de transmissão. Algumas DST são assintomáticas. Assim, os indivíduos procuram os serviços de saúde apenas na presença de sintomas, perpetuando a cadeia de disseminação, reforçando a importância do rastreamento para o controle dessas doenças e prevenção de infecção pelo HIV ${ }^{40}$. Segundo estudiosos, na presença de DST, o risco de transmissão do HIV é quatro a cinco vezes maior, mostrando a relevância deste fator de risco no ciclo de even$\operatorname{tos}^{14,32,40}$. Valendo ressaltar que no grupo de adolescentes e adultos jovens a iniciação sexual precoce contribui para a alta vulnerabilidade às doenças sexualmente transmissíveis e HIV/AIDS ${ }^{41}$.

No quesito acessibilidade ao serviço, verificou-se que a maior parte dos indivíduos deste estudo residia no município e na zona urbana, o que pode ter facilitado o acesso à atenção e informação, contrário às populações que residem na zona rural e em outros municípios. Diante desta realidade surge a necessidade de descentralização destas ações de prevenção, controle e tratamento do HIV/AIDS para municípios menores, carentes e com dificuldade de acesso aos serviços de saúde, como forma de viabilizar a inclusão no sistema de saúde, conforme os princípios e as diretrizes universais do Sistema Único de Saúde/ SUS, garantindo desta forma eficiência, universalidade e resolutividade no atendimento. A resposta social direcionada à prevenção ao HIV/ AIDS depende diretamente do acesso dos indivíduos à informação, assim como aos recursos disponíveis para sua proteção, promovidos pelos setores, representados pelas Instituições de referência, face ao controle dos importantes problemas de saúde, na busca da redução das desigualdades e distribuição equitativa do poder, nos diferentes contextos sociais ${ }^{35,42}$.

Nesta pesquisa ficou enfatizado que características sociodemográficas e comportamentais de jovens e respectivos parceiros, especialmente em união estável, podem ter impacto na transmissão da infecção e doença, constituindo aspectos de extrema relevância para o planejamento e a execução de ações em serviços. No aspecto individual, a noção de vulnerabilidade, está relacionada ao comportamento e hábitos de vida, diante da possibilidade de infectar-se ou adoecer. Assim, considera-se fundamental a sensibilização para mudança de atitude de jovens, face à epidemia da AIDS, incentivando o comportamento de auto- proteção, cujo impacto contribui para a situação de saúde e mudança do perfil da infecção e doença nesse grupo populacional. Ressalta-se a importância do levantamento e consolidação de indicadores que podem subsidiar políticas e práticas eficazes de prevenção e controle dos principais fatores de exposição, contribuindo assim para o controle da infecção, disseminação da doença e mudanças no quadro epidemiológico da AIDS.

Para finalizar, torna-se indispensável considerar as limitações da pesquisa, que por se tratar de um estudo de corte transversal não permite estabelecer nexo causal. O estudo se limitou a analisar jovens registrados no Centro de Referência DST-HIV-AIDS para triagem sorológica ou tratamento, referenciados de outros serviços ou por demanda espontânea; não podendo atribuir os resultados da pesquisa à população de Feira de Santana e municípios circunvizinhos. Entretanto, destaca-se a importância de estudos desta natureza que buscam consolidar os dados do serviço, ação de grande relevância para a consolidação de indicadores direcionados ao Sistema Nacional de Informação.

\section{Conclusão}

No aspecto individual, a noção de vulnerabilidade está relacionada ao comportamento e aos hábitos de vida, diante da possibilidade de infectarse ou adoecer. De acordo com os objetivos deste estudo verificou-se a relação entre a infecção por HIV e o uso de outras drogas e orientação homossexual/bissexual entre homens, e o uso de álcool e outras drogas e união estável para as mulheres, e o parceiro usuário de drogas e/ou portador de HIV e DST para ambos os sexos. Assim, considera-se fundamental a sensibilização para uma mudança de atitude entre adolescentes e adultos jovens frente à epidemia da AIDS, incentivando o comportamento de autoproteção, cujo impacto contribui para a situação de saúde e mudança do perfil da infecção e doença nesse grupo populacional.

Ressalta-se a importância do levantamento e consolidação de indicadores que podem subsidiar políticas e práticas eficazes de prevenção e controle dos principais fatores de exposição, contribuindo assim para mudanças no quadro epidemiológico da AIDS, na adolescência e juventude. 


\section{Colaboradores}

BS Pereira, VS Sampaio, CAL Silva, HS Costa, MTR Amaral e MCO Costa participaram igualmente de todas as etapas de elaboração do artigo.

\section{Referências}

1. Unaids - Brasil. Report on the global AIDS epidemic. Joint United Nations Programme on HIV/AIDS. [página na Internet] 2012. [acessado $2013 \mathrm{dez} 25]$. Disponível em: http://www.unaids.org/en/

2. Santos SMJ, Rodrigues JA, Carneiro WS. Doenças sexualmente transmissíveis: conhecimento de alunos do ensino médio. DST $j$ bras doenças sex transm 2009; 21(2):63-68.

3. Taquette SR. Epidemia de HIV/Aids em adolescentes no Brasil e na França: semelhanças e diferenças. Saude soc 2013; 22(2):618-628.

4. Gupta GR, Ogden J, Warner A. Moving forward on women's gender-related HIV vulnerability: The good news, the bad news and what to do about it. Glob Public Health 2011; 6(Supl. 3):S370-S382.

5. Brasil. Ministério da Saúde (MS). Secretaria de Vigilância em Saúde. Departamento de DST, Aids e Hepatites Virais. Recomendações para a Atenção Integral a Adolescentes e Jovens Vivendo com HIV/Aids. 1 $1^{\text {a }}$ Edição. Brasília: MS; 2013.

6. Unaids - Brasil. A ONU e a resposta à aids no Brasil. documento conjunto dos agencias as Nações Unidas no Brasil, que foi elaborado pelo escritório do UNAIDS em cooperação com o Escritório dor Coordenador Residente, ACNUR, UNODC, UNICEF, UNIFEM, UNFPA, UNESCO,OPAS/OMS, OIT e PNUD. 2a Edição. Brasília: Unaids-Brasil; 2010.

7. Brasil. Ministério de Saúde (MS). Departamento de DST e Aids e Hepatites Virais. Portal sobre aids, doenças sexualmente transmissíveis e hepatites virais. [página na Internet]. [acessado 2013 fev 7]. Disponível em: www.aids.gov.br

8. Organização Mundial da Saúde (OMS). Riesgos para la salud de los jóvenes. Geneva: OMS; 2011. [Nota descritiva, $n^{\circ} 345$ ].

9. Moura RF, Souza CBJ, Almeida PC. Adesão de adolescentes de um serviço de saúde de Fortaleza ao uso de condom e fatores associados. Cienc Cuid Saude 2009; 8(1):11-18.

10. Gubert D, Madureira VSF. Iniciação sexual de homens adolescentes. Cien Saude Colet 2008; 13(Supl. 2):2247-2256

11. Brasil. Ministério da Saúde (MS). Secretaria de Atenção a Saúde. Programa Nacional de DST/Aids. Boletim Epidemiológico Aids e DST. Brasília: MS; 2012.

12. Costa MCO, Santos BC, Souza KEP, Cruz NLA, Santana MC, Nascimento OC. HIV/AIDS e Sífilis em Gestantes Adolescentes e Adultos Jovens: Fatores de Exposição e Risco dos Atendimentos de um Programa em DST/HIV/AIDS na Rede Pública de Saúde/SUS, Bahia, Brasil. Revista Baiana de Saúde Pública 2011; 35(Supl. 1):179-185.

13. Camargo BV, Giacomozzi AI, Wachelke JFR, Aguiar A. Relações Amorosas, Comportamento Sexual e Vulnerabilidade de Adolescentes Afrodescendentes e Brancos em Relação ao HIV/aids. Saude soc 2010; 19(Supl. 2):36-50.

14. Taquette SR, Matos HJ, Rodrigues AO, Bortolotti LR, Amorim E. A epidemia de AIDS em adolescentes de 13 a 19 anos, no município do Rio de Janeiro: descrição espaço-temporal. Revista da Sociedade Brasileira de Medicina Tropical 2011; 44(4):467-470. 
15. Araújo TM, Monteiro CFS, Mesquita GV, Alves ELM, Carvalho KM, Monteiro RM. Fatores de risco para infecção por HIV em Adolescentes. Rev enferm UERJ 2012; 20(2):242-247.

16. Tavares CM, Schor N, França JI, Diniz SG. Factors associated with sexual initiation and condom use among adolescents on Santiago Island, Cape Verde, West Africa. Cad Saude Publica 2009, 25(9):19691980.

17. Custódio G, Massuti AM, Trevisol FS, Trevisol DJ. Comportamento Sexual e de Risco para DST e gravidez na adolescência. DST - J bras Doenças Sex Transm 2009; 21(2):60-64.

18. Bustos FA, Elías FD, Bertolini PR. Conducta sexual en adolescentes varones: haciaunnuevo horizonte. Rev Anacem 2011; 5(2):123-127.

19. Instituto Brasileiro de Geografia e Estatística (IBGE). Censo demográfico 2013. Rio de Janeiro: IBGE; 2013.

20. Centers for Disease Control and Prevention (CDC). HIV among Youth. National Center for HIV/AIDS, Viral Hepatitis, STD, and TB Prevention, CDC. USA: CDC; 2011.

21. Meira MBV. Sobre estruturas etárias e ritos de passagem. Ponto-e-vírgula 2009; 5:185-201.

22. Taquete SR. Feminização da Aids e adolescência. Adolesc Saúde 2009; 6(1):33-40.

23. Brito AM, Castilho EA, Szwarcwald CL. AIDS e infecção pelo HIV no Brasil: uma epidemia multifacetada. Rev Soc Bras Med Trop 2001; 34(2):207217.

24. Vilela MP, Brito TRB, Goyatá SLT, Arantes CIS Perfil epidemiológico dos usuários do Centro de Testagem e Aconselhamento de Alfenas, Minas Gerais. Rev Eletr Enf 2010; 12(2):327-330.

25. Schneider IJC, Ribeiro C, Breda D, Skalinski LC, D’órsi E. Perfil epidemiológico dos usuários dos Centros de Testagem e Aconselhamento do Estado de Santa Catarina, Brasil, no ano de 2005. Cad Saude Publica. 2008; 24(7):326-330.

26. Taquete Stella R. Feminização da Aids e adolescência. Adolesc Saúde 2009; 6(1):33-40.

27. Lazarini FM, Melchior R, Gonzalez AD, Matsuo T. Tendência da epidemia de casos de aids no Sul do Brasil no período de 1986 a 2008. Rev Saude Publica 2012; 46(6):960-968.

28. Bertakis KD, Azari R, Helms LJ, Callahan EJ, Robbins JA. Gender Differences in the Utilization of Health Care Services. J Fam Pract 2000; 49(2):147152.

29. Takahashi RF, Shima H, Souza M. Mulher e AIDS: perfil de uma população infectada e reflexões sobre suas implicações sociais. Rev Latino Am Enfermagem 1998; 6(5):59-65.

30. Dourado I, Vera MA, Barreira D, Brito AM. Tendência da epidemia de AIDS no Brasil após a terapia antiretroviral. Rev Saude Publica 2006; 40(Supl.): 9-17.
31. Gupta GR, Ogden J, Warner A. Moving forward on women's gender-related HIV vulnerability: The good news, the bad news and what to do about it. Glob Public Health 2011; 6(Supl. 3):370-382.

32. Taquette SR, Vilhena MM, Paula MC. Doenças sexualmente transmissíveis na adolescência: estudo de fatores de risco. Rev Soc Bras Med Trop 2004; 37(3):210-214.

33. Camargo EAI, Ferrari RAP. Adolescentes: conhecimentos sobre sexualidade antes e após a participação em oficinas de prevenção. Cien Saude Colet 2009; 14(3):937-946.

34. Higgins JA, Hoffman S, Dworkin SL, Rethinking G. Heterosexual Men, and Women's Vulnerability to HIV/AIDS. Am J Public Health 2010; 100(3):435-445.

35. Paiva V, Pupo LR, Barboza R. O direito à prevenção e os desafios da redução da vulnerabilidade ao HIV no Brasil. Rev Saude Publica 2006; 40(Supl.):109-119.

36. Anjos RD, Silva JAS, Val LF, Rincon LA, Nichiata LYI. Diferenças entre adolescentes do sexo feminino e masculino na vulnerabilidade individual ao HIV. Rev esc enferm USP 2012; 46(4):829-837.

37. Tudiver F, Myers T, Kurtz RG, Orr K, Rowe C, Jackson E, Bullock SL. The Talking Sex Project : Results of a Randomized Controlled Trial of Small-Group AIDS Education for 612 Gay and Bisexual Men. Eval Health Prof 1992; 15(1):26-42.

38. Folch C, Casabona J, Munõz R, Gonzales V, Zaragoza $\mathrm{K}$. Incremento en la prevalencia del VIH y en las conductas de riesgo asociadas en hombres que tienen sexo con hombres: 12 años de encuestas de vigilancia conductual en Cataluña. Gac Sanit 2010; 24(1):40-46.

39. Centers for Disease Control and Prevention (CDC). STD Trends in the United States: 2011 National Data for Chlamydia, Gonorrhea, and Syphilis. National Center for HIV/AIDS, Viral Hepatitis, STD, and TB Prevention, CDC. USA: CDC; 2012.

40. Codes JS, Cohen DA, Melo NA, Teixeira GG, Leal AS, Silva TJ, Oliveira MPR. Detecção de doenças sexualmente transmissíveis em ambientes clínicos e não clínicos na Cidade de Salvador, Bahia, Brasil. Cad Saude Publica 2006; 22(2):325-334.

41. Gubert D, Madureira VSF. Iniciação sexual de homens adolescentes. Cien Saude Colet 2008; 13(Supl. 2):2247-2256

42. Ayres JRCM. Práticas educativas e prevenção de HIV/Aids: lições aprendidas e desafios atuais. Interface (Botucatu) 2002; 6(11):11-24.

Artigo apresentado em 03/09/2013

Aprovado em 09/10/2013

Versão final apresentada em 23/10/2013 